\title{
Long-term reparative effects of mesenchymal stem cell therapy following neonatal hyperoxia-induced lung injury
}

\author{
Ronald P. Sutsko', Karen C. Young 1,2, Ana Ribeiro', Eneida Torres' ${ }^{1}$, Myra Rodriguez', Dorothy Hehre', Carlos Devia', \\ lan McNiece $^{2}$ and Cleide Suguihara'
}

BACKGROUND: Mesenchymal stem cell (MSC) therapy may prevent neonatal hyperoxia-induced lung injury (HILI). There are, however, no clear data on the therapeutic efficacy of MSC therapy in established HILI, the duration of the reparative effects, and the exact mechanisms of repair. The main objective of this study was to evaluate whether the long-term reparative effects of a single intratracheal (IT) dose of MSCs or MSC-conditioned medium (CM) are comparable in established HILI.

METHODS: Newborn rats exposed to normoxia or hyperoxia from postnatal day (P)2)-P16 were randomized to receive IT MSCs, IT CM, or IT placebo (PL) on P9. Alveolarization and angiogenesis were evaluated at P16, P30, and P100.

RESULTS: At all time periods, there were marked improvements in alveolar and vascular development in hyperoxic pups treated with MSCs or CM as compared with PL. This was associated with decreased expression of inflammatory mediators and an upregulation of angiogenic factors. Of note, at P100, the improvements were more substantial with MSCs as compared with CM.

CONCLUSION: These data suggest that acute effects of MSC therapy in HILI are mainly paracrine mediated; however, optimum long-term improvement following HILI requires treatment with the MSCs themselves or potentially repetitive administration of CM.

$\mathbf{H}^{2}$ yperoxic exposure of the developing lung is one of the main contributing factors to the development of bronchopulmonary dysplasia (BPD) (1). This disease affects $\sim 40 \%$ of extremely low birth weight infants (2) and is characterized by disordered microvasculature development and decreased alveolarization (3). In its most severe form, BPD is complicated by severe pulmonary hypertension $(\mathrm{PH})$, and long-term survivors have deranged lung function and neurodevelopmental impairment (1). Indeed, although severe BPD affects $<10 \%$ of extremely low birth weight preterm infants, effective therapies must be generated given that this disease is a significant cause of mortality in these patients.

One of the new potential strategies for the treatment of BPD is cell-based therapy. Cell-based therapies have now proven to be efficacious in the repair of several organs, including heart, lung, and brain (4). Since the seminal report by Krause and colleagues in 2001, demonstrating that a single bone marrow-derived stem cell could engraft in the lung and adopt the phenotype of airway epithelial cells (5), several reports have demonstrated that stem cells can improve lung injury induced by bacterial pneumonia (6), endotoxin (7), and hyperoxia (8).

One of the most utilized stem cell populations in preclinical and clinical regenerative medicine is the mesenchymal stem cell (MSC). These stem cells are easily expanded, readily accessible, and immunoprivileged (9). Furthermore, their anti-inflammatory and proangiogenic properties (9) make them attractive therapeutic entities for neonatal hyperoxia-induced lung injury (HILI). In fact, two recent reports have demonstrated the efficacy of MSCs in preventing neonatal HILI. A single intratracheal (IT) or intravenous dose of MSCs was shown to prevent the parenchymal and vascular damage observed in neonatal HILI $(8,10)$, albeit the effects appeared to be mainly paracrine mediated (10). It is, however, not known if MSC therapy could abrogate the lung damage evidenced in established neonatal HILI and whether the reparative effects, if any, would be sustained in the long term. In addition, because recent data suggest that the mechanism of MSC repair is mainly paracrine mediated, we sought to test the hypothesis that the administration of a single dose of IT MSCs or a single dose of IT MSC-conditioned medium (CM) to neonatal rats with established HILI would yield comparable lung repair both acutely and in the long term.

To address these questions, the current study evaluated the use of IT MSCs and IT CM to treat established HILI in newborn rats, an animal model of severe BPD (11). We show that the administration of a single IT dose of MSCs or CM to newborn rats with established HILI similarly improves lung injury in the short term. However, in refute of our hypothesis, we show that more significant long-term repair following HILI is obtained following a single IT dose of MSCs as compared with a single IT dose of CM. The current findings therefore suggest that the administration of actual MSCs themselves and not just a CM cocktail is necessary to optimize long-term lung repair following neonatal HILI.

\section{RESULTS}

Acute Improvement in HILI Following Intratracheal Administration of MSCs or CM

Following IT administration of placebo (PL), MSCs, or CM, newborn pups were evaluated at postnatal day $(\mathrm{P}) 16(n=18$ litters) and P30 ( $n=16$ litters). Hyperoxia-exposed newborn

'Department of Pediatrics/Division of Neonatology, University of Miami Miller School of Medicine, Miami, Florida; ${ }^{2}$ Department of Medicine/Interdisciplinary Stem Cell Institute, University of Miami Miller School of Medicine, Miami, Florida. Correspondence: Cleide Suguihara (csuguihara@med.miami.edu) 
rats treated with IT PL had alveolar "simplification" at P16 similar to that seen in BPD (12). This was evidenced by fewer alveoli with large diameters and decreased alveolar septation (Figure 1a). These histological changes persisted and worsened even after a 2-wk recovery in room air (RA) (P30). Remarkably, administration of a single dose of IT MSCs or CM at P9 dramatically improved the alveolar structure in the hyperoxic pups at both P16 and P30. This was evidenced by an attenuation of the increase in mean linear intercept and average alveolar area in the treated pups (Figure $\mathbf{1 b} \mathbf{b}-\mathbf{e}$ ). There was no difference in alveolarization between the MSC and CM treatment groups.

Hyperoxic PL-treated rats also exhibited capillary rarefaction, as evidenced by decreased Von Willebrand Factor staining (Figure 2a) and a 60\% decrease in the vascular density as compared with normoxia. In contrast, treatment with MSCs or CM improved vascular density at both P16 (Figure 2b) and P30 (Figure 2c), and the MSC- or CM-treated hyperoxic animals had twice as many vessels as those treated with PL. There was no significant difference between the MSC and CM groups. Of note, this improvement in vascularization was

a
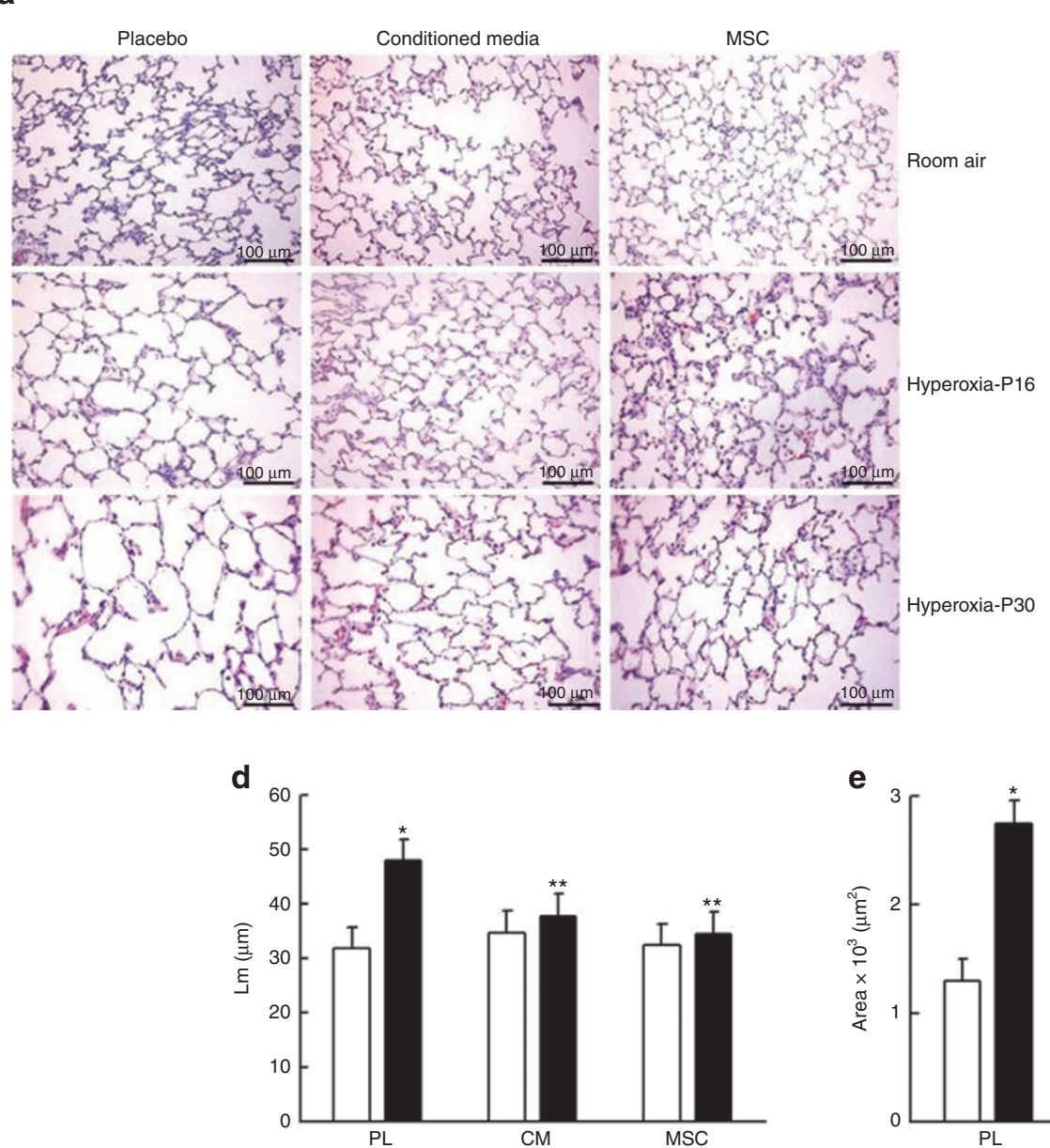

associated with increased expression of the angiogenic factors vascular endothelial growth factor (VEGF) and angiopoeitin-1 in hyperoxic animals treated with MSCs or CM (Figure 2d,e).

Chronic hyperoxia also produced significant $\mathrm{PH}$ and rightventricular hypertrophy in PL-treated animals, as evidenced by increased right-ventricular systolic pressure (RVSP) and right ventricle/left ventricle + septum $(S)$ ratio. However, treatment of hyperoxic animals with MSCs markedly attenuated these changes at P16 (Figure 3a,b) and P30 (Figure 3c,d). In contrast to the MSC-treated group, administration of CM improved these measures of PH at P16 but not at P30 (Figure 3a-d).

Finally, in accordance with the known immunomodulatory properties of MSCs (9), the acute reparative effects of MSCs and CM in neonatal rats with HILI were associated with a decreased gene expression of the proinflammatory cytokines interleukin-6 (IL-6) and IL-1 $\beta$ (Figure 4a,b). Moreover, MSCs and CM significantly limited the hyperoxia-induced downregulation of thyroid transcription factor (TTF-1) (Figure 4c), a transcription factor shown to be important in lung morphogenesis and the control of surfactant protein production (13).
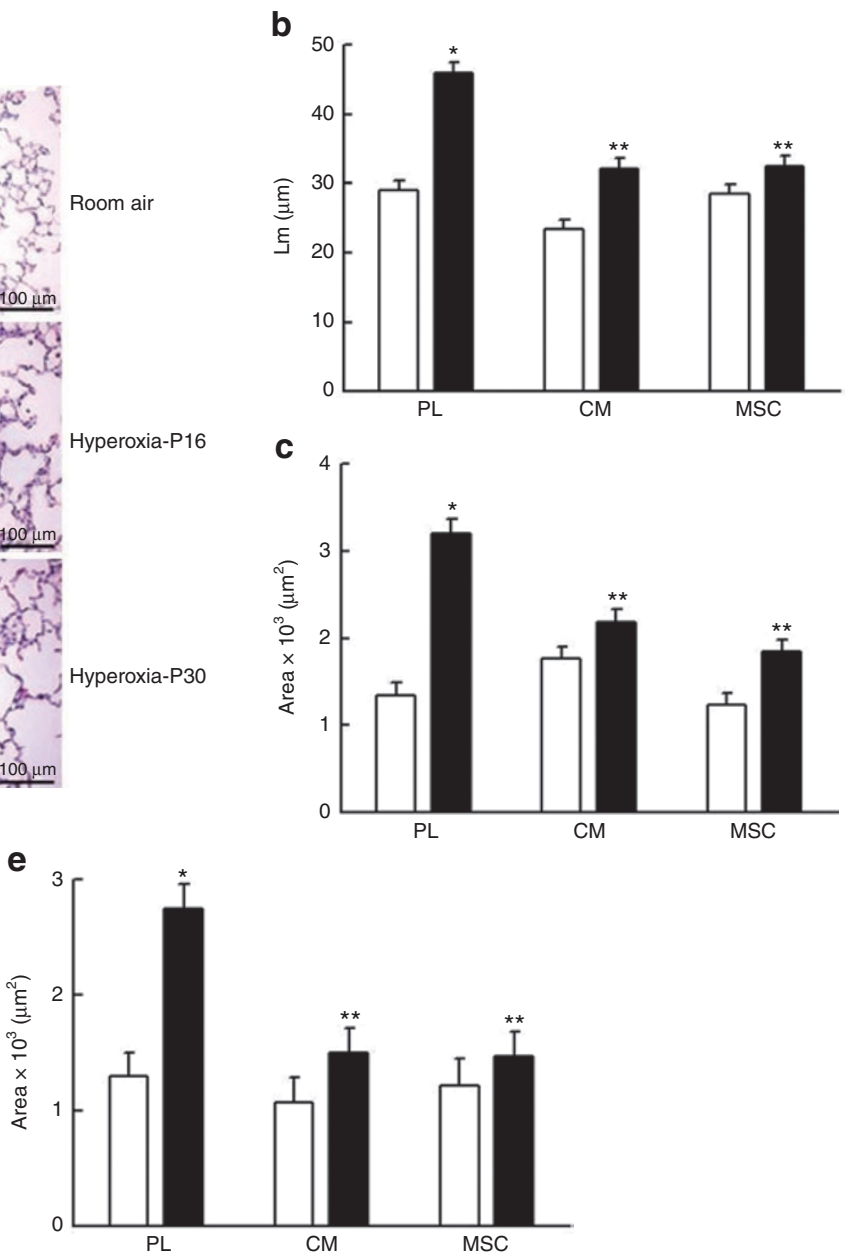

Figure 1. Improvement in alveolarization following intratracheal mesenchymal stem cells/MSC-conditioned medium (IT MSC/CM) at P16 and P30. (a) Hematoxylin and eosin-stained lung sections demonstrating improved alveolar structure in hyperoxia-exposed pups treated with (IT MSC/CM) at P16 and P30. Original magnification $\times 200$. Bars $=100 \mu \mathrm{m}$. IT MSC/CM attenuated the increase in (b) mean linear intercept $(\mathrm{Lm})$ and $(\mathbf{c})$ average alveolar area (AAA) observed in hyperoxic placebo-treated (Hyp PL) pups at P16. (d) Similar changes in Lm and (e) AAA were seen at P30 ( ${ }^{*} P<0.001$ room air (RA) vs. Hyp PL, ${ }^{* * P}<0.001$ Hyp PL vs. Hyp MSC or Hyp CM; $n=5$ /group). White bars are RA and black bars are hyperoxia. 
a
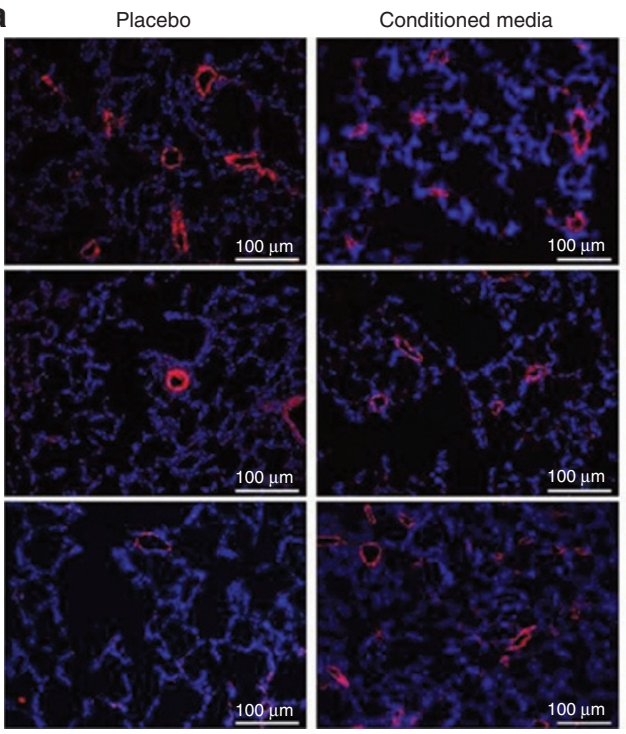

d

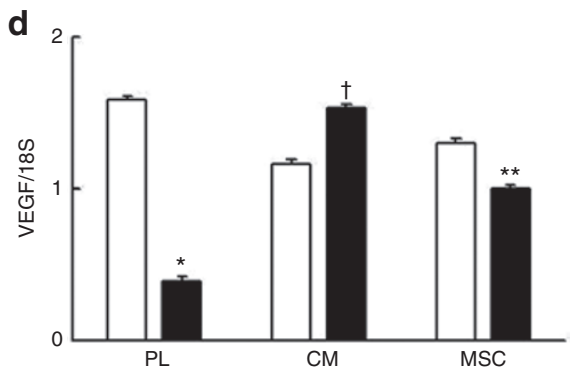

MSC

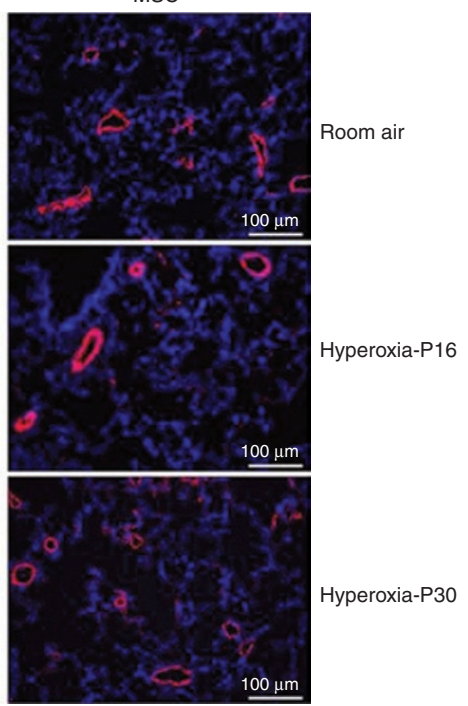

b
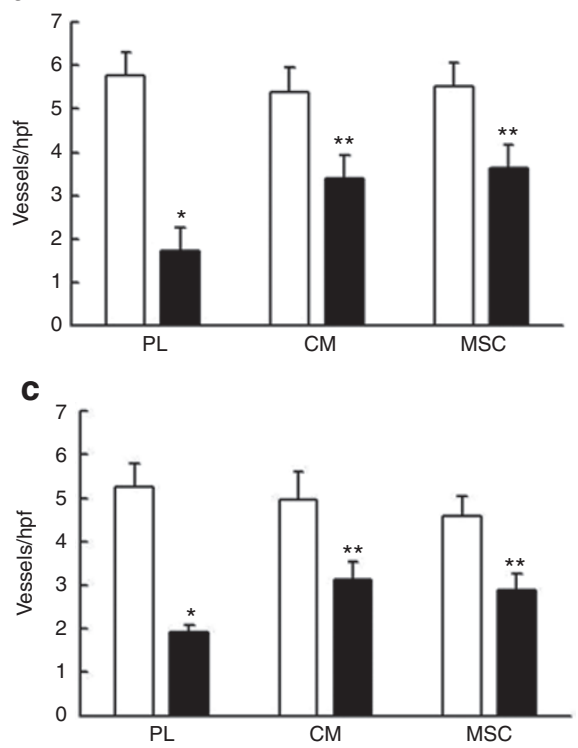

e

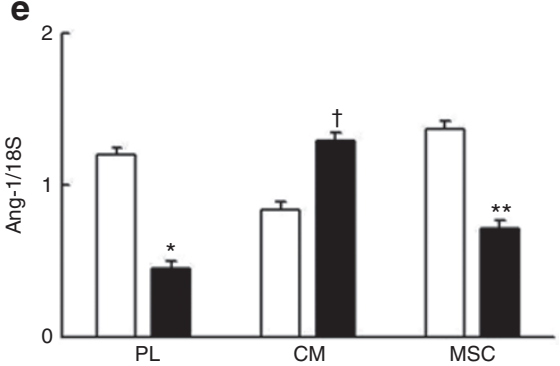

Figure 2. Improvement in lung vascular density following intratracheal mesenchymal stem cells/MSC-conditioned medium (IT MSC/CM) at P16 and P30. (a) Lung sections stained with Von Willebrand Factor (red) and 4' 6-diamidino-2-phenylindole (blue) demonstrating improved vascular density in hyperoxia-exposed pups treated with (IT MSC/CM) at P16 and P30. Original magnification $\times 200$. Bars $=100 \mu \mathrm{m}$. IT MSC/CM increased the number of vessels $(20-50 \mu \mathrm{m}) /$ high power field at (b) P16 and (c) P30 (*P $<0.001$ room air (RA) vs. Hyperoxic placebo (Hyp PL), ${ }^{* *}<0.04$ Hyp PL vs. Hyp MSC or Hyp CM; $n=$ 5/group). These findings were associated with a relative increase in the gene expression of (d) VEGF and (e) Ang-1 in the hyperoxic IT MSC/CM groups at P16, ${ }^{*} P<0.001$ RA vs. Hyp PL, ${ }^{* *} P<0.002$ Hyp PL vs. Hyp MSC, ${ }^{+} P<0.001$ Hyp PL vs. Hyp CM; $n=7 /$ group). White bars are RA and black bars are hyperoxia. Ang-1, angiopoietin-1; VEGF, vascular endothelial growth factor.

\section{Long-Term Improvement in HILI Following Intratracheal MSC Therapy}

To elucidate whether a single dose of IT MSCs or CM would provide sustained recovery following HILI, animals were also evaluated after a 12 -wk recovery period in RA (P100; $n=4$ litters). Even after this long recovery period, the lungs from PL-treated hyperoxia animals had strikingly significant alveolar simplification (Figure 5a), evidenced by increased mean linear intercept and average alveolar area (Figure $5 b, c)$. In contrast, these morphometric findings were significantly decreased in the hyperoxic rats that were treated with MSCs or CM (Figure $5 b, c$ ). The magnitude of this improvement was greater in the groups treated with MSCs as compared with those treated with CM.

Consistent with the above changes, PL-treated hyperoxic animals, recovering in RA for $12 \mathrm{wk}$, continued to have a diminished vascular density (Figure 6a). In contrast, the administration of MSCs or CM attenuated the impairment in vascular development(Figure 6b). However, the degree of improvement was significantly greater in those animals treated with MSCs as compared with those treated with CM.
Similarly, after 12 wk of recovery in RA, PL-treated hyperoxic rats continued to have a significant increase in $\mathrm{PH}$ evidenced by an elevation in RVSP (Figure 6c). The administration of IT MSCs significantly limited the elevation of RVSP, but treatment with CM did not produce any improvement in RVSP as compared with PL. Neither IT MSCs nor CM improved the degree of right-ventricular hypertrophy (Figure 6d).

\section{Engraftment}

Lung sections from all groups ( $n=3$ animals/group) were immunostained with surfactant protein SP-C and antigreen fluorescent protein (GFP) antibody (Figure 7). Engrafted $\mathrm{GFP}^{\text {pos }}$ cells that express SP-C were manually counted in five random fields per section and expressed as a percentage of all nuclei present in that field. The percentage of GFP ${ }^{\text {pos }}$ cells in the lungs at P16, P30, and P100 in the hyperoxia-exposed pups were $0.3 \pm 0.11 \%, 0.3 \pm 0.08 \%$, and $0.4 \pm 0.15 \%$, respectively. Of these GFP ${ }^{\text {os }}$ cells, the percentages that expressed SP-C at $\mathrm{P} 16, \mathrm{P} 30$, and $\mathrm{P} 100$ were $13 \pm 11 \%, 75 \pm 22 \%$, and $77 \pm 10 \%$ respectively. 

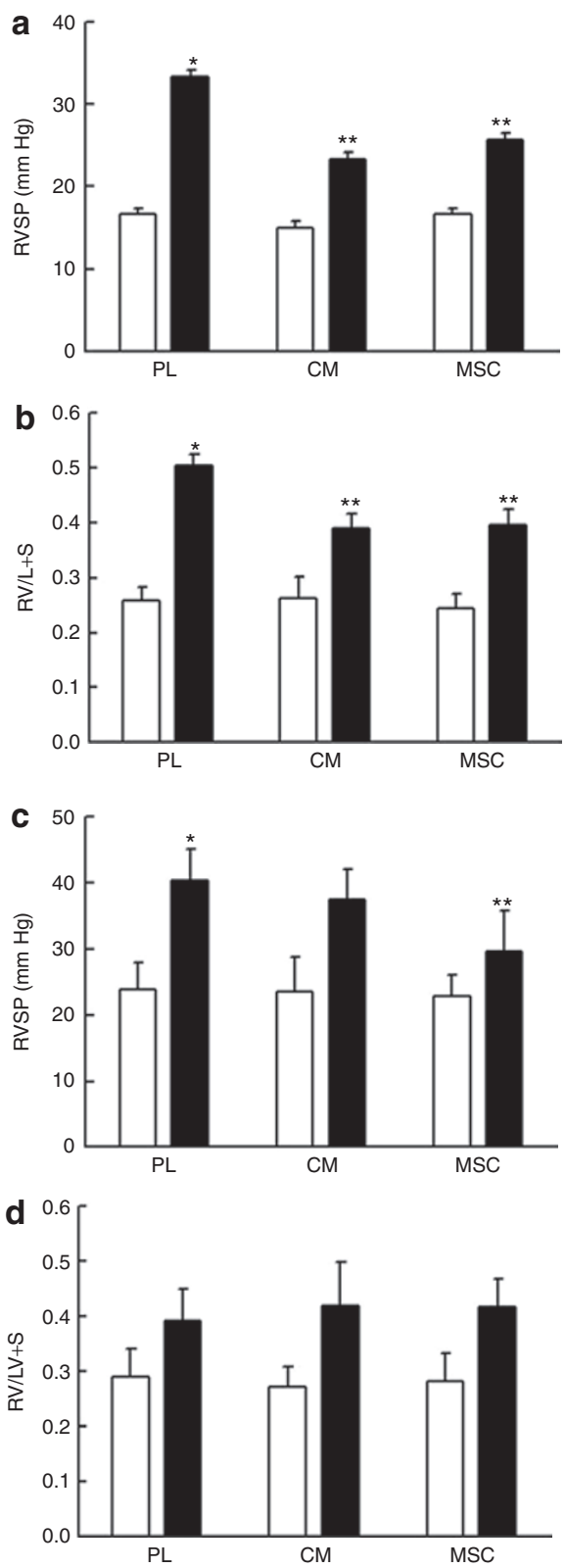

Figure 3. Improvement in pulmonary hypertension following intratracheal mesenchymal stem cells/MSC-conditioned medium (IT MSC/CM) at P16 and P30. (a) Administration of (IT MSC/CM) to hyperoxic pups attenuated the increase in right-ventricular systolic pressure (RVSP) $\left({ }^{*} P<0.001\right.$ room air (RA) vs. hyperoxic placebo (Hyp PL), ${ }^{* *} P<0.001$ Hyp PL vs. Hyp MSC or Hyp CM; $n=20-28 /$ group) and (b) right ventricle/left ventricle (RV/LV) + septum (S) $\left({ }^{*} P<0.001\right.$ RA vs. Hyp $P L$, ${ }^{* *} P<0.03$ Hyp PL vs. Hyp MSC or Hyp CM; $n=8-19$ /group), in Hyp PL pups at P16. (c) At P30, only IT MSC attenuated the increase in RVSP $\left({ }^{*} P<0.001\right.$ RA vs. Hyp $P L$, ${ }^{* *} P<0.001$ Hyp PL vs. Hyp MSC; $n=10-14 /$ group). (d) IT MSC/CM did not affect RV/LV + S at P30. White bars are RA and black bars are hyperoxia.

\section{DISCUSSION}

This study demonstrates that the administration of a single IT dose of MSCs or CM to neonatal rats with established HILI comparably improves alveolarization, angiogenesis, and $\mathrm{PH}$ in the short term, but the administration of IT MSCs provides more substantial long-term repair in neonatal rats recovering from HILI. These results suggest that although the acute
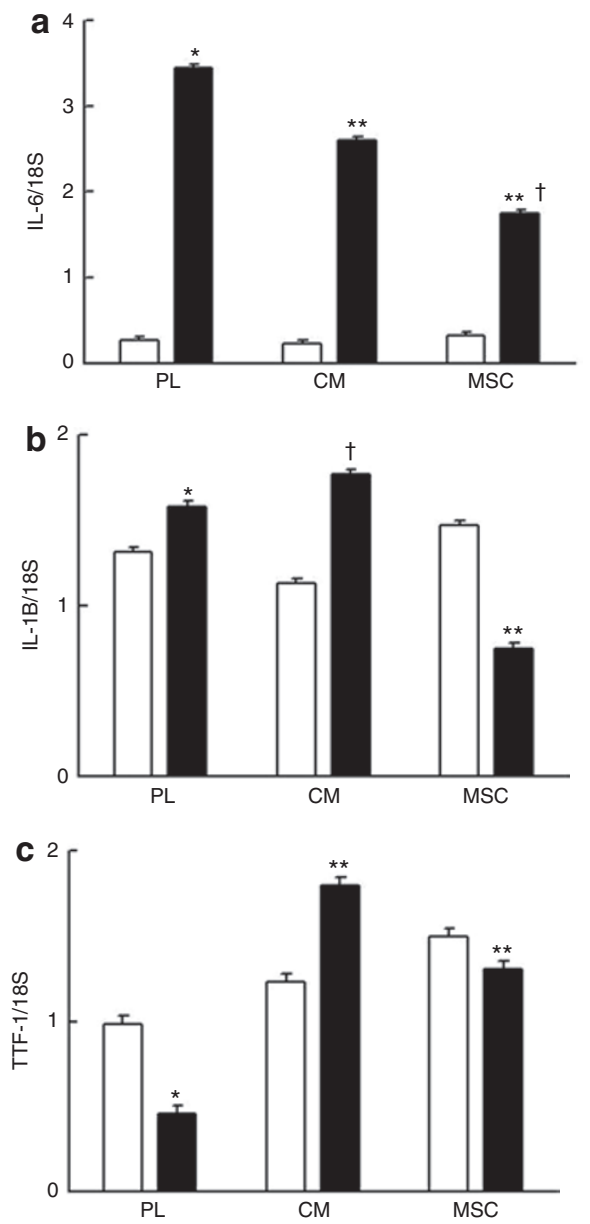

Figure 4. Acute downregulation of lung inflammatory genes following intratracheal mesenchymal stem cells (IT MSC). (a) IT MSC/MSC-conditioned medium (MSC/CM) downregulated the gene expression of interleukin (IL)-6 $\left({ }^{*} P<0.001\right.$ room air (RA) vs. hyperoxic placebo (Hyp PL), ${ }^{* *} P<0.001$ Hyp PL vs. Hyp MSC or Hyp CM, ${ }^{+} P<0.001$ Hyp MSC vs. Hyp CM, $n=7 /$ group). (b) IT MSC decreased the expression of II- $1 \beta\left({ }^{*} P<0.001\right.$ RA vs. Hyp PL, ${ }^{*} P<0.001$ Hyp PL vs. Hyp MSC; $n=7 /$ group) whereas IT CM increased II-1 $\beta$ expression ( ${ }^{+} P<0.001$ Hyp PL vs. Hyp CM; $n=7 /$ group). (c) IT MSC/CM limited the downregulation of thyroid transcription factor-1 (TTF-1) in Hyp PL pups $\left({ }^{*} P<0.001\right.$ RA vs. Hyp PL, ${ }^{* *} P<0.001$ Hyp PL vs. Hyp MSC or Hyp $\mathrm{CM}, n=7$ group). White bars are RA and black bars are hyperoxia.

improvement in HILI following MSC therapy may be mainly paracrine mediated, optimum long-term recovery requires the presence of the MSCs themselves. This is an important finding given that it provides further insight into the potential of IT MSC therapy to provide sustained improvement in severe $\mathrm{BPD}$, and opens the door for further evaluation of the paracrine factors released from MSCs that may mediate the acute improvement in HILI.

Cell therapy has now emerged as one of the new strategies for the treatment of severe BPD $(8,10)$. In fact, several recent preclinical studies have demonstrated improvement in neonatal HILI following the administration of bone marrow-derived stem cells $(8,10)$. In a seminal study by van Haaften and colleagues, intratracheal prophylactic delivery of MSCs to neonatal rats prevented hyperoxia-induced arrest of alveolar and vascular development, and improved survival and exercise 


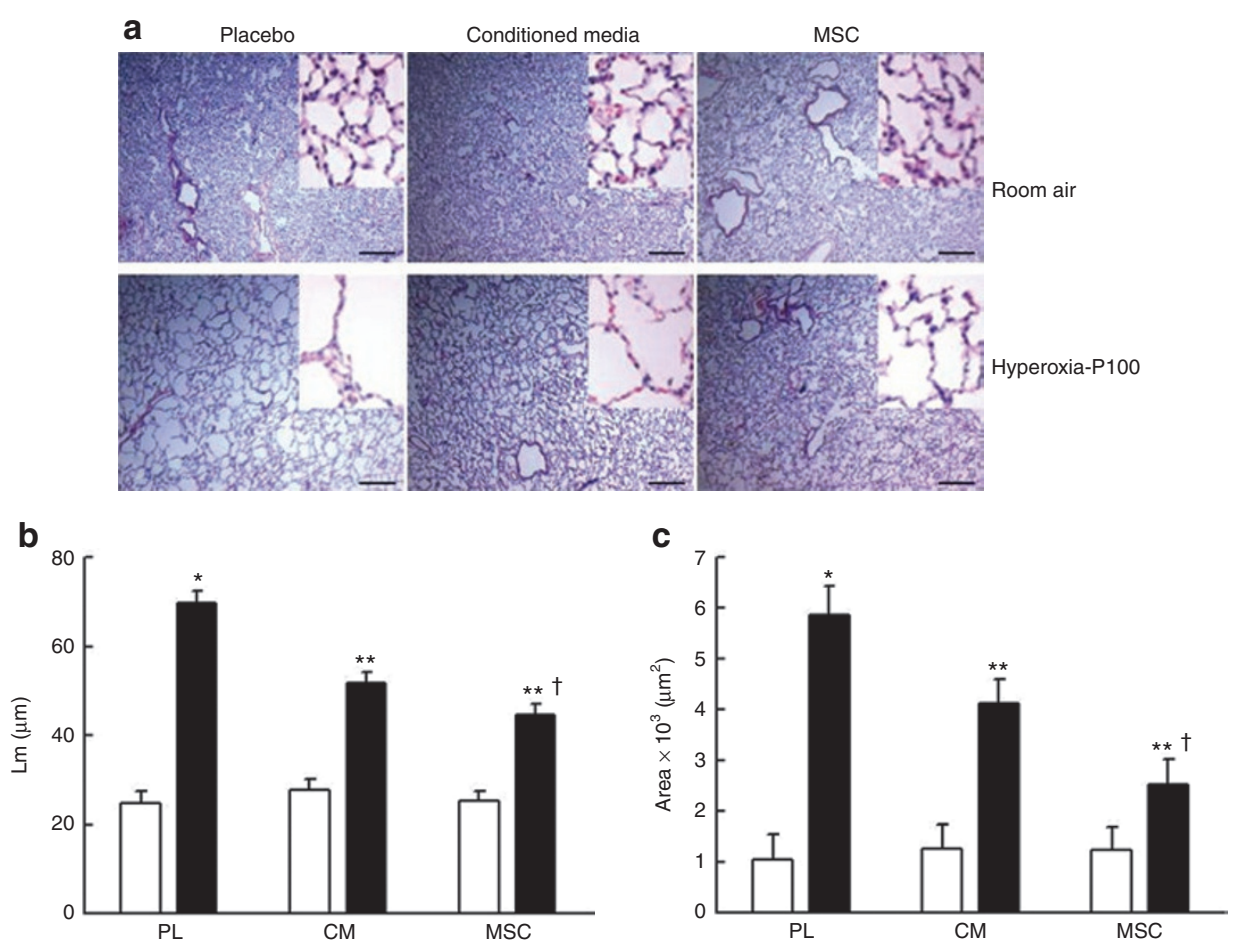

Figure 5. Long-term improvement in alveolarization following intratracheal mesenchymal stem cells/MSC-conditioned medium (IT MSC/CM). (a) Hematoxylin and eosin-stained lung sections demonstrating improved alveolar structure in hyperoxic pups treated with (IT MSC) and CM at P100. Shown in magnification of $\times 25$ and $\times 100$. Bars $=500 \mu \mathrm{m}$. (b) IT MSC/CM attenuated the increase in mean linear intercept $\left({ }^{*} P<0.001\right.$ room air (RA) vs. hyperoxic placebo (Hyp PL), $* * P$ $<0.001$ Hyp PL vs. Hyp MSC or Hyp CM, ${ }^{+} P<0.04$ Hyp MSC vs. Hyp CM; $n=5 /$ group), and (c) average alveolar area $\left(* P<0.001\right.$ RA vs. Hyp PL, ${ }^{* *} P<0.03$ Hyp PL vs. Hyp MSC or Hyp CM, ${ }^{+} P<0.03$ Hyp MSC vs. Hyp CM; $n=5 /$ group), observed in Hyp PL pups at P100, but these improvements were more in the MSC group. White bars are RA and black bars are hyperoxia.
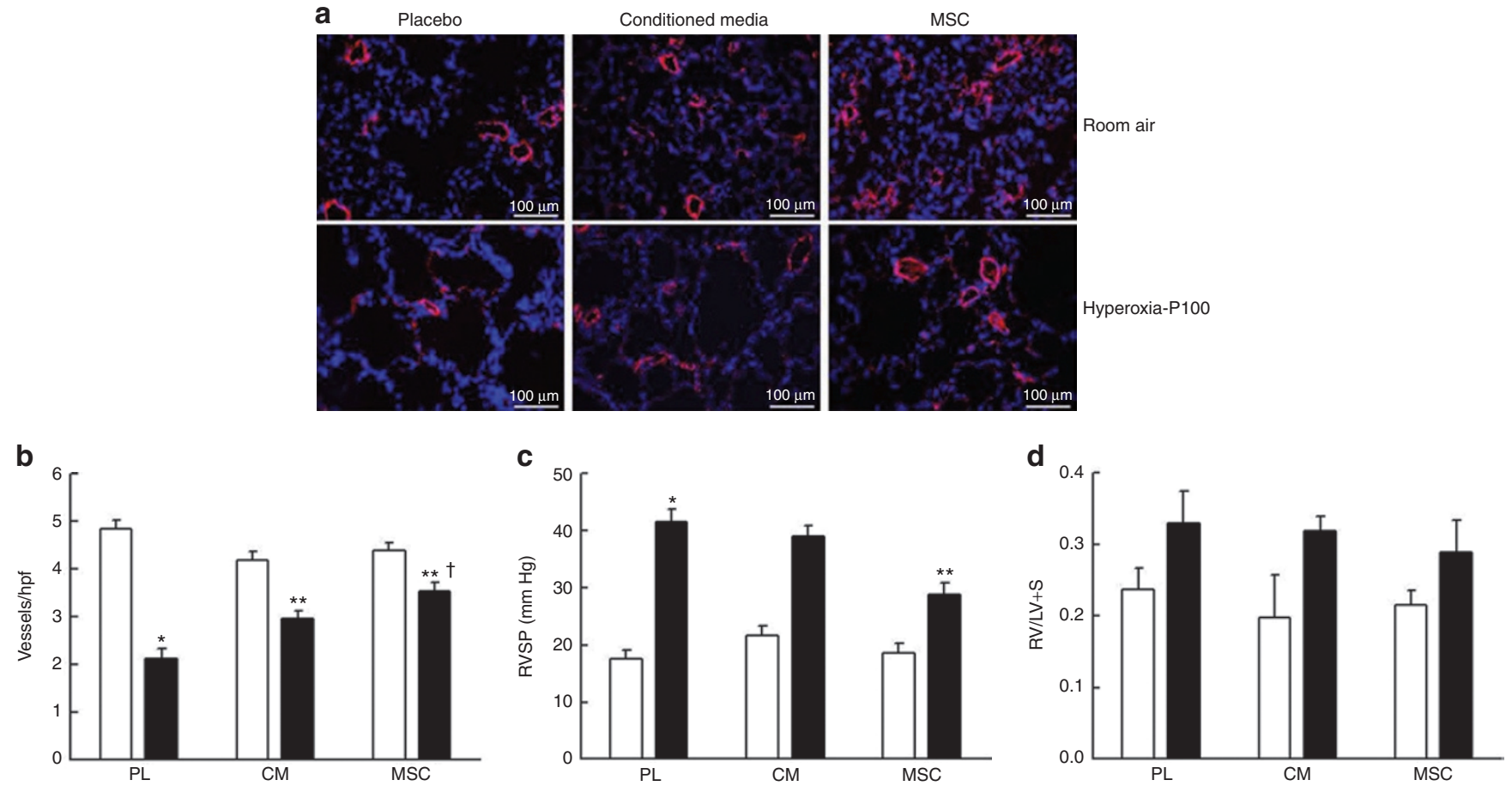

Figure 6. Long-term improvement in lung vascular density and pulmonary hypertension following intratracheal mesenchymal stem cells/MSC-conditioned medium (IT MSC/CM). (a) Lung sections stained with Von Willebrand Factor (red) and 4' 6-diamidino-2-phenylindole (blue) demonstrating improved vascular density in hyperoxia-exposed pups treated with (IT MSC/CM) at P100. Original magnification $\times 200$. Bars $=100 \mu \mathrm{m}$. (b) IT MSC/CM increased the vascular density at P100 $\left({ }^{*} P<0.001\right.$ room air (RA) vs. hyperoxic placebo (Hyp PL), ${ }^{*} P<0.005$ Hyp PL vs. Hyp MSC or Hyp CM; $n=5 /$ group), but the effect of MSCs was more substantial $\left({ }^{+} P<0.04\right.$ Hyp MSC vs. Hyp CM). (c) IT MSC attenuated the increase in right-ventricular systolic pressure observed in Hyp PL pups at P100 $\left({ }^{*} P<0.001 \mathrm{RA}\right.$ vs. Hyp PL, ${ }^{*} P<0.002$ Hyp PL vs. Hyp MSC; $n=8$ /group). (d) Right-ventricular hypertrophy was unaffected by IT MSC/CM at P100. White bars are RA and black bars are hyperoxia. 

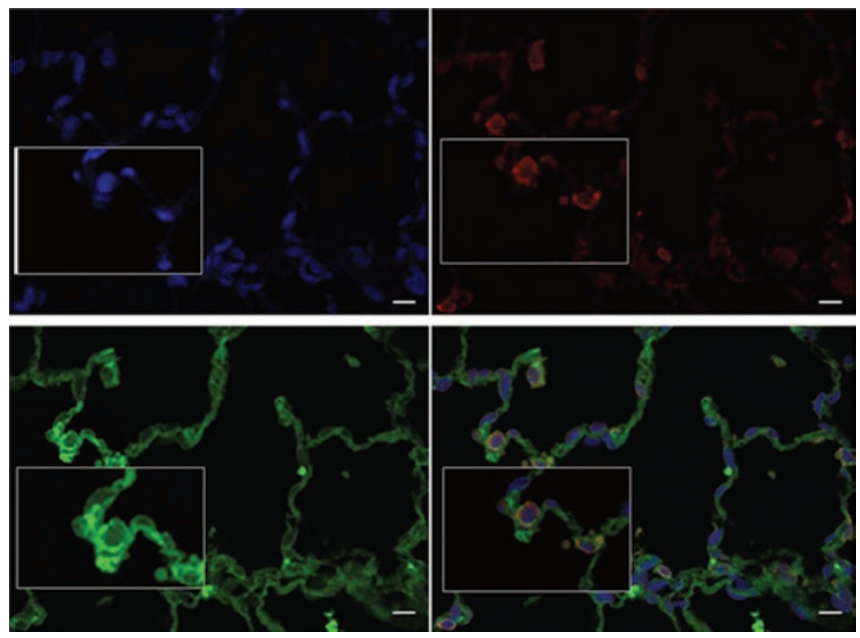

Figure 7. Lung engraftment of intratracheal mesenchymal stem cells (IT MSC). Lung sections stained with 4' 6-diamidino-2-phenylindole (blue), green fluorescent protein (GFP) (green), SP-C (red), and GFP+SP-C (yellow) demonstrating engraftment of GFPpos MSC in hyperoxic pups treated with intratracheal MSC at P16. Original magnification $\times 400$. Bar $=10 \mu \mathrm{m}$.

tolerance (8). Of note, MSC-derived conditioned media prevented hyperoxia-induced alveolar epithelial cell apoptosis and enhanced endothelial cord formation, suggesting that the mechanism of action of MSCs to prevent HILI is mainly paracrine mediated (8). This concept has since been supported by Aslam et al., who showed that intravenous administration of MSCs also prevented HILI in neonatal mice but the administration of intravenous $\mathrm{CM}$ had a more pronounced reparative effect acutely (10).

In contrast to these aforementioned reports that explored the protective effects of MSCs in HILI, our study provides new data showing that a single dose of IT MSCs or CM comparably improves alveolarization, angiogenesis, and $\mathrm{PH}$ in neonatal rats with established HILI but only in the short term. Indeed, in our current study, we chose to administer the MSC therapy $7 \mathrm{~d}$ after hyperoxia exposure, an interval at which there is a marked arrest of alveolar and vascular development as well as PH (11). Thus, our current study adds to the literature by showing that IT MSC therapy can be used as a therapeutic strategy for established neonatal HILI. Moreover, it shows that the effects of IT MSCs in HILI are mainly paracrine mediated, at least acutely, given that IT CM had comparable short-term reparative effects.

This is a plausible mechanism of action given that striking benefits of MSC therapy have been documented despite low engraftment rates, and furthermore, MSCs are known to secrete several potent growth and immunomodulatory factors (14). Among these secreted factors, VEGF and angiopoietin-1 have been shown to be important in lung development and repair (15). In our current study, we show that the acute improvement in alveolarization and lung vascular development following IT MSC or CM administration to neonatal rats with HILI was associated with a marked increase in the expression of VEGF and angiopoietin-1, suggesting that the improvement in HILI may be secondary to improved angiogenesis stemming from the replenishment of these vasculogenic growth factors.
In addition to the MSC- and CM-associated increase in the expression of the aforementioned angiogenic factors in the hyperoxic-injured lung, our study also provides further evidence of the anti-inflammatory effects of MSCs. We show that IT MSCs or CM significantly suppress the hyperoxia-induced increase in the expression of IL- 6 and IL-8. These findings are supportive of the findings by Aslam et al. showing decreased bronchoalveolar lavage fluid neutrophil and macrophage count following intravenous MSC administration in neonatal mice with HILI (10). This anti-inflammatory property is attractive to harness in HILI given that proinflammatory cytokines such as Il- $1 \beta$ and IL- 6 are known to be increased in the bronchoalveolar lavage fluid of infants with BPD (16), and furthermore, the overexpression of cytokines during alveolarization has been shown to result in the development of "emphysema" in mice $(3,17)$.

Another interesting finding in the current study is the MSC- or CM-associated increase in the expression of TTF-1 in hyperoxic-injured lungs. We show that neonatal PL-treated rats with HILI had decreased expression of this transcription factor, but a single dose of IT MSCs or CM almost completely restored its expression to baseline levels. TTF-1 is known to be involved in the regulation of lung morphogenesis and influences the genes responsible for vasculogenesis and inflammation (18). TTF-1 knockout mice are known to have significant disruption of lung morphogenesis (19), and increased localized TTF-1 expression has been demonstrated in regions of regeneration in postmortem human lungs with BPD, suggesting that it is critical in alveolar restoration during recovery from neonatal lung diseases (13). Our current findings therefore suggest that the MSC/CM-associated modulation of TTF-1 expression may be important acutely in lung regeneration following HILI.

In accordance with these findings that suggest a paracrinemediated mechanism of MSC repair, (at least in the short term), absence of any significant lung engraftment of GFPlabeled MSCs at 1, 3, or $13 \mathrm{wk}$ after IT administration was not surprising, a finding consistent with previously published data describing engraftment rates of $0-5 \%(8,10)$. However, it should be noted that the insignificant engraftment in our current study and the published literature should be interpreted with caution, given that GFP is unstable, engrafted cells may lose their fluorescence, and there can be variability of expression even among similar cell types. Thus, it is plausible that despite the inability to document significant retention of the IT administered MSCs, the cells could still be present within the lung structure.

This latter theory could potentially explain the disparity in the long-term reparative effects of IT CM and IT MSCs seen in our current study. Indeed, in contrast to the similar acute effects, our long-term study shows that the administration of a single IT dose of MSCs at P9 improved alveolarization and angiogenesis to a more marked degree as compared with IT CM, when pups with established HILI were evaluated at P100. This suggests that the MSCs themselves are more efficacious as compared with CM for long-term repair of the hyperoxicinjured lung and suggests a possible direct cell-cell mediated effect. This is a distinct possibility, given that direct cell-cell 
interactions of the MSCs may partially restore the endogenous stem cell pool, leading to increased genesis of alveolar cells, thereby augmenting the repair process. This generation of lung stem cells may explain how low levels of MSC engraftment in the lung can still produce a beneficial effect.

Nonetheless, the possibility that the long-term reparative effects of MSCs following HILI may actually be also paracrine mediated should not be overlooked. Theoretically, the disparity in the longterm therapeutic effects of the IT MSCs and IT CM may be due to the fact that once the factors within the CM are consumed by the damaged lung tissue, then the rate of lung recovery lessens because the CM contains only a finite amount of factors. If in fact this theory is accurate, then it is possible that the mechanism of MSC repair may be paracrine and the presence of the cells provides a source for the long-term secretion of reparative factors. Thus, theoretically serial repetitive dosing of IT CM may actually produce equivalent long-term repair as a single dose of IT MSC.

Finally, it is important to mention the limitations of this study. The histopathological changes evidenced in this model are more in accordance with severe BPD, an entity that is less frequently observed currently. It would be crucial to evaluate the effects of MSCs in rodent models with less oxygen exposure. In addition, in our evaluation of the long-term effects of MSCs in HILI, during the recovery period in which the pups were in normoxia it is quite possible that they may have been hypoxemic and thus the lung morphological changes seen at P100 could be secondary to mixed hypoxic and hyperoxic insults on the alveolar and vasculature structures. However, it is also notable that in our study a large percentage of the GFPpos cells retained in the lung also expressed SP-C at 3 and $13 \mathrm{wk}$ after IT administration of MSCs. The potential of MSCs to differentiate into alveolar epithelial cells has been previously demonstrated in vitro (8). Moreover, a similar degree of SP-C/GFP colocalization was demonstrated by van Haaften et al. following IT administration of GFPpos MSCs to neonatal rats with HILI, but admittedly, the approach of using dual labeling to document the degree of in vivo engraftment and differentiation has limitations (8). The close juxtaposition of GFPpos cells with alveolar epithelial cells or cell fusion may give the false impression of cell differentiation. We also did not evaluate all the possible harmful effects of the MSC therapy. Of note, we did not demonstrate any worsening in pulmonary vascular remodeling following the MSC therapy. Further studies will need to be performed to evaluate the possible formation of lung sarcomas and other adverse effects.

In conclusion, the present study provides new information on the potential of a single dose of IT MSCs to provide longterm repair of the neonatal hyperoxic-injured lung. We show that although IT MSCs and CM acutely improve established neonatal HILI, the IT administration of MSCs conveys more significant long-term repair. The implications of the present findings are tremendous given that they suggest that the acute effects of MSC are mainly paracrine mediated, and thus administration of a cocktail of factors secreted by the MSCs could improve HILI (at least acutely). Admittedly, although the long-term effects of MSCs in HILI may also be partially paracrine mediated, in order to achieve long-term sustained repair, a single IT administration of MSCs is superior to a single IT dose of CM. Further studies will need to be performed to ascertain if the long-term effects are secondary to important cell-cell mediated interactions or if repetitive dosing of CM will produce equivalent long-term repair.

\section{METHODS \\ Animals}

Adult male GFP transgenic and pregnant female Sprague Dawley rats were purchased from The Rat Resource and Research Center (Columbia, MO). Animals were treated according to National Institutes of Health guidelines, and the protocol was approved by the Animal Care and Use Committee of the University of Miami Miller School of Medicine.

\section{MSC Retrieval}

MSCs obtained from male GFPpos Sprague Dawley rats were isolated and cultured as previously described (20) (see Supplementary Methods online). For injection the cells were thawed, washed with phosphate buffered saline, and resuspended in phosphate buffered saline at $2 \times 10^{6}$ cells $/ 50 \mu$ l. CM was prepared as previously described (10) and a single IT dose of $50 \mu \mathrm{l}$ was utilized for administration.

\section{Experimental Procedure}

Randomly chosen pups from 38 litters of Sprague Dawley rats were assigned at birth to RA or hyperoxia $\left(90 \% \mathrm{O}_{2}\right)$ from P2 to P16. Pups were housed in a plexiglass chamber with $\mathrm{O}_{2}$ monitoring. Litters for each experimental group were limited to 10 pups to control for the effect of litter size on nutrition and growth. Due to a mortality rate of $<10 \%$ in the hyperoxia-exposed pups, studies were repeated with several litters in order to obtain equivalent numbers of pups per experimental condition. Mothers were rotated every $48 \mathrm{~h}$ to standardize the nutrition provided to each litter. Oxygen exposure was continuous, with brief interruptions for animal care $(<10 \mathrm{~min} /$ day $)$. After $2 \mathrm{wk}$ in the designated exposure, the litters were removed and placed in RA. One subset of the animals was studied for hemodynamic measurements and morphometry at this time, whereas other groups were allowed to recover in RA for an additional 2 or $12 \mathrm{wk}$ before being studied.

\section{MSC Administration}

On P9, the pups were anesthetized with ketamine $(30 \mathrm{mg} / \mathrm{kg})$ and xylazine $(4 \mathrm{mg} / \mathrm{kg})$ via i.p. injection. The trachea was exposed through a small incision on the midline of the neck, and MSCs $\left(2 \times 10^{6}\right.$ in $\left.50 \mu \mathrm{l}\right)$, $\mathrm{CM}(50 \mu \mathrm{l})$, or phosphate buffered saline/PL $(50 \mu \mathrm{l})$ was delivered by tracheal puncture with a 30-gauge needle. Pups were exposed to continuous bypass flow of RA or oxygen via rodent nose cone throughout the procedure, and for recovery, they were placed into a warmed plastic chamber under normoxia or hyperoxia conditions. Once pups were fully awake, they were placed back with their dams.

\section{Hemodynamic Measurements}

RVSP was evaluated as previously described (21). Briefly, a thoracotomy was performed and a 22-gauge needle connected to a pressure transducer was inserted into the right ventricle. RVSP was measured and recorded on a Gould polygraph (model TA-400; Gould instruments, Cleveland, $\mathrm{OH}$ ).

\section{Right-Ventricular Hypertrophy}

Right-ventricular hypertrophy was assessed as previously described (22). Briefly, the right ventricle was dissected from the left ventricle and $\mathrm{S}$, and all were weighed. The ratio between the weights of right ventricle and left ventricle $+\mathrm{S}$ was calculated.

\section{Lung Alveolarization}

Pulmonary vascular morphometry was performed as previously described (23) (Supplementary Methods online). Serial 5- $\mu$ m-thick paraffin-embedded sections obtained from the lungs were stained with hematoxylin and eosin. Images from six randomly selected, nonoverlapping parenchymal fields were acquired from one lung section of each 
animal (5-6/group) at 20× magnification. Care was taken to exclude bronchioles, vessels, and ambiguous objects, ensuring that only alveoli were included in the study. Images were captured by a blinded observer, and the mean linear intercept, a measure of interalveolar wall distance, and the average alveolar area, a measure of air space enlargement, were analyzed using Metamorph Imaging System software (Molecular Devices, Sunnyvale, CA) as previously described (24).

\section{Lung Vascular Density}

Vascular density was evaluated as previously described (14). Briefly, lung sections were stained with polyclonal rabbit antihuman Von Willebrand Factor (Dako, Carpinteria, CA), a marker of endothelial cells, and 4' 6-diamidino-2-phenylindole, a marker of cell nuclei. Six randomly selected, nonoverlapping parenchymal fields were evaluated from lung sections of each animal (5-6/group). The number of blood vessels $(20-50 \mu \mathrm{m}$ in diameter) in each high-power field was counted by a blinded observer.

\section{Quantitative Real-Time PCR}

The gene expression of IL-1 $\beta$, IL-6, angiopoietin-1, VEGF, and TTF- 1 was quantified by real-time reverse transcriptase PCR using SuperArray (Frederick, MD). The relative quantity of IL-1 $\beta$, IL-6, angiopoietin-1, VEGF, and TTF-1 was normalized to $18 \mathrm{~S}$ expression.

\section{Assessment of Engraftment and Differentiation}

Serial 5 - $\mu \mathrm{m}$ paraffin-embedded sections obtained from the upper and lower lobes of the lungs were dewaxed and rehydrated in descending grades of alcohol. Following antigen retrieval and blocking of nonspecific binding sites with a protein blocker, the lung sections were stained with the type II alveolar specific marker, antisurfactant protein C (1:50; AB3786; Millipore, Billerica, MA); anti-Alexa Fluor IgG (1:200; AF 594, A21207, Life Technologies, Carlsbad, CA); and antiGFP (1:50; sc-5385 AF488, Santa Cruz Biotechnology, Santa Cruz, CA) antibodies. Engrafted GFP ${ }^{\text {pos }}$ cells expressing SP-C were imaged with a confocal microscope (Leica DMI 6000, Mannheim, Germany) and five random fields per section were counted by a blinded observer and expressed as a percentage of all nuclei present in that field (details in Supplementary Methods online).

\section{Statistical Analysis}

Data are expressed as mean \pm SEM and were analyzed by two-way ANOVA with post hoc analysis (Holm-Sidak). $P$ values $<0.05$ were considered statistically significant. Statistical analysis was performed using SigmaStat software (SyStat Software, San Jose, CA).

\section{SUPPLEMENTARY MATERIAL}

Supplementary material is linked to the online version of the paper at http:// www.nature.com/pr

\section{STATEMENT OF FINANCIAL SUPPORT}

This work was supported by the following grants: University of Miami, Project New Born (to C.S., K.C.Y.); Ikaria 2008-2009 Advancing Newborn Medicine Grant program (to R.P.S.); National Institutes of Health K08 Award; Florida Biomedical Research Award; and Batchelor Research Foundation Award (to K.C.Y.).

\section{REFERENCES}

1. Jobe AH, Bancalari E. Bronchopulmonary dysplasia. Am J Respir Crit Care Med 2001;163:1723-9.

2. Stoll BJ, Hansen NI, Bell EF, et al. Neonatal outcomes of extremely preterm infants from the NICHD Neonatal Research Network. Pediatrics 2010;126:443-56.

3. Jobe AJ. The new BPD: an arrest of lung development. Pediatr Res 1999;46:641-3.
4. Weiss DJ, Kolls JK, Ortiz LA, Panoskaltsis-Mortari A, Prockop DJ. Stem cells and cell therapies in lung biology and lung diseases. Proc Am Thorac Soc 2008;5:637-67.

5. Krause DS, Theise ND, Collector MI, et al. Multi-organ, multi-lineage engraftment by a single bone marrow-derived stem cell. Cell 2001;105:369-77.

6. Krasnodembskaya A, Song Y, Fang X, et al. Antibacterial effect of human mesenchymal stem cells is mediated in part from secretion of the antimicrobial peptide LL-37. Stem Cells 2010;28:2229-38.

7. Gupta N, Su X, Popov B, Lee JW, Serikov V, Matthay MA. Intrapulmonary delivery of bone marrow-derived mesenchymal stem cells improves survival and attenuates endotoxin-induced acute lung injury in mice. J Immunol 2007;179:1855-63.

8. van Haaften T, Byrne R, Bonnet S, et al. Airway delivery of mesenchymal stem cells prevents arrested alveolar growth in neonatal lung injury in rats. Am J Respir Crit Care Med 2009;180:1131-42.

9. Phinney DG, Prockop DJ. Concise review: mesenchymal stem/multipotent stromal cells: the state of transdifferentiation and modes of tissue repair-current views. Stem Cells 2007;25:2896-902.

10. Aslam M, Baveja R, Liang OD, et al. Bone marrow stromal cells attenuate lung injury in a murine model of neonatal chronic lung disease. Am J Respir Crit Care Med 2009;180:1122-30.

11. Roberts RJ, Weesner KM, Bucher JR. Oxygen-induced alterations in lung vascular development in the newborn rat. Pediatr Res 1983;17:368-75.

12. Wilson WL, Mullen M, Olley PM, Rabinovitch M. Hyperoxia-induced pulmonary vascular and lung abnormalities in young rats and potential for recovery. Pediatr Res 1985;19:1059-67.

13. Stahlman MT, Gray ME, Whitsett JA. Expression of thyroid transcription factor-1(TTF-1) in fetal and neonatal human lung. J Histochem Cytochem 1996;44:673-8.

14. Lee JW, Fang X, Krasnodembskaya A, Howard JP, Matthay MA. Concise review: mesenchymal stem cells for acute lung injury: role of paracrine soluble factors. Stem Cells 2011;29:913-9.

15. Thébaud B. Angiogenesis in lung development, injury and repair: implications for chronic lung disease of prematurity. Neonatology 2007;91:291-7.

16. Kotecha S, Wilson L, Wangoo A, Silverman M, Shaw RJ. Increase in interleukin (IL)-1 beta and IL-6 in bronchoalveolar lavage fluid obtained from infants with chronic lung disease of prematurity. Pediatr Res 1996;40:250-6.

17. Ray P, Tang W, Wang P, et al. Regulated overexpression of interleukin 11 in the lung. Use to dissociate development-dependent and -independent phenotypes. J Clin Invest 1997;100:2501-11.

18. Maeda Y, Davé V, Whitsett JA. Transcriptional control of lung morphogenesis. Physiol Rev 2007;87:219-44.

19. Kimura S, Hara Y, Pineau T, et al. The T/ebp null mouse: thyroid-specific enhancer-binding protein is essential for the organogenesis of the thyroid, lung, ventral forebrain, and pituitary. Genes Dev 1996;10:60-9.

20. Dominici M, Le Blanc K, Mueller I, et al. Minimal criteria for defining multipotent mesenchymal stromal cells. The International Society for Cellular Therapy position statement. Cytotherapy 2006;8:315-7.

21. Yet SF, Perrella MA, Layne MD, et al. Hypoxia induces severe right ventricular dilatation and infarction in heme oxygenase-1 null mice. J Clin Invest 1999;103:R23-9.

22. Fulton RM, Hutchinson EC, Jones AM. Ventricular weight in cardiac hypertrophy. Br Heart J 1952;14:413-20.

23. Young KC, Torres E, Hatzistergos KE, Hehre D, Suguihara C, Hare JM. Inhibition of the SDF-1/CXCR4 axis attenuates neonatal hypoxia-induced pulmonary hypertension. Circ Res 2009;104:1293-301.

24. McGrath-Morrow SA, Cho C, Soutiere S, Mitzner W, Tuder R. The effect of neonatal hyperoxia on the lung of p21Waf1/Cip1/Sdi1-deficient mice. Am J Respir Cell Mol Biol 2004;30:635-40. 